\title{
Confirmation and Analysis of Fentanyl and Its Analogues Without the Standards
}

\author{
Wang Chengyun $^{1}$, Zhang Weiya ${ }^{1}$, Yan Jie ${ }^{1,}$, , Lin Junfeng ${ }^{2}$, Xie Tangtang ${ }^{2}$, Yang Zuojun ${ }^{2}$ \\ ${ }^{1}$ The Testing and Technology Center for Industrial Products, Shenzhen Custom, Shenzhen, P. R. China \\ ${ }^{2}$ Shenzhen Academy of Inspection and Quarantine, Shenzhen, P. R. China \\ Email address: \\ wangchengyun2009@126.com (Wang Chengyun),zwyciq@163.com (Zhang Weiya), szyanj163@163.com (Yan Jie), \\ jflin1000@163.com (Lin Junfeng), tangtangxie@139.com (Xie Tangtang),869419981@qq.com (Yang Zuojun) \\ ${ }^{*}$ Corresponding author
}

\section{To cite this article:}

Wang Chengyun, Zhang Weiya, Yan Jie, Lin Junfeng, Xie Tangtang, Yang Zuojun. Confirmation and Analysis of Fentanyl and Its Analogues Without the Standards. American Journal of Chemical Engineering. Vol. 7, No. 1, 2019, pp. 43-50. doi: 10.11648/j.ajche.20190701.15

Received: March 11, 2019; Accepted: May 22, 2019; Published: June 15, 2019

\begin{abstract}
In recent years, the number of controlled fentanyl species has increased gradually, and up to now, the number of controlled fentanyl species increased to twenty-seven. Since fentanyl standards are difficult to obtain, there are only several kinds of controlled fentanyl species involved in the literature, and the analysis of all 27 controlled fentanyl species have not been reported in the literature. Therefore, it is of great significance to establish a method for the rapid screening and confirmation of all 27 controlled fentanyl species without the use of standards. In this paper, an analytical method for the rapid screening and confirmation of 27 controlled fentanyl substances without the use of standards was established. Suspected samples were rapidly screened by ultra-high performance liquid chromatography/Orbitrap high resolution mass spectrometry (UPLC/Orbitrap HRMS). Qualitative identification was performed through the quasi-molecular ion accurate masses of target compounds. Confirmation was carried out by secondary ion segments. It is the first time that all of 27 controlled fentanyl substances was tested. The proposed method was simple, rapid and accurate, applicable for the rapid screening and confirmation of 27 controlled fentanyl substances without standards.
\end{abstract}

Keywords: Fentanyl, UPLC/Orbitrap HRMS, Screening

\section{Introduction}

Since 2000, the global reporting of the abuse of new psychoactive substances (NPS) has increased dramatically [1] Between 2008 and 2015, 102 countries reported 644 NPS to the United Nations Office on Drugs and Crime. NPS is a single substance or mixture that is not controlled by the UN international conventions but is harmful to public health. It is a drug analogue that the criminals have obtained to chemically modify the controlled drugs in order to escape the blow of law enforcement agencies. NPS has the similar or stronger effect of controlled drugs and has become the third-generation drug that is globally popular after traditional drugs and synthetic drugs, generally including synthetic cannabinoids, stimulants (such as phenethylamine, cathinone, piperazine, etc.), magic agents or isolated hallucinogens (ketamine and phencyclidine), tranquilizers, tryptamine, aminoguanidine and fentanyl synthetic opioids [2]. The Belgian pharmacologist and pharmaceutical chemist Paul Janssen developed fentanyl in 1960. It was at the time a revolutionary compound with a potency much greater than that of the natural opioid morphine, and a significantly greater safety margin than other pharmaceutical synthetic opioids such as dextromoramide, meperidine and phenoperidine. Under Janssen's leadership, a deeper understanding of the structure/activity relationships led to the development of the very potent analgesics sufentanil, alfentanil, lofentanil and carfentanil, which could also be used for anaesthesia. These drugs have been successfully used clinically for over fifty years, and have made possible complex surgeries and the successful management of chronic pain for millions of patients worldwide. Fentanyl, together with codeine, methadone and morphine are four opioid analgesics on the World Health Organization's (WHO) List of Essential Medicines [3]. In California, United States in 1979, a series of deaths occurred 
in people who injected drugs that were subsequently attributed to an analogue of fentanyl, $\alpha$-methylfentanyl. Initially discovered and patented by Janssen, $\alpha$-methylfentanyl had neither been evaluated in humans for safety or efficacy, nor approved as a pharmaceutical drug. This was the first recorded instance of a completely novel clandestinely synthesized opioid, and earned the sobriquet "designer drug". Through the early 1980s, $\alpha$-methylfentanyl was quickly joined by a series of other fentanyl analogues including para- fluorofentanyl, 3-methylfentanyl, $\beta$-hydroxyl fentanyl, and a number of others that were identified in deaths in the west coast of the United States. Subsequently, the use of fentanyl itself became widespread due to its availability worldwide in injectable, sublingual and transdermal forms, and it also became subject to diversion and abuse. At the beginning of 2014, the availability of fentanyl in the United States had started to increase exponentially [4], with crime laboratories reporting more than 4,500 fentanyl cases in 2014 and over 14,400 in 2015. Around the same time, a group of fentanyl new psychoactive substances (NPS) began to appear in the street drug market, beginning with acetylfentanyl, and butyrylfentanyl. In view of the abuse and addiction of fentanyl substances, international organizations have restricted their use and the number of restricted species increase gradually. Fentanyl was first controlled under the 1961 Single Convention on Narcotic Drugs in 1964. Sufentanyl and alfentanyl were then controlled in 1980 and 1984, respectively. Acetyl- $\alpha$-methylfentyl, $\alpha$-methylfentanyl and 3-methylfentanyl were controlled in 1988. Thiofentanyl, $\alpha$-methyl-thiofentanyl, $\quad \beta$-hydroxy fentanyl, 3-methylthiofentanyl, $\beta$-hydroxy-3- methylfentanyl, para-fluorofentanyl were controlled in 1990. Remifentanil was controlled in 1999, while acetylfentanyl and ocfentanyl were controlled in 2013. Butyrfentanyl and furanfentanyl were controlled in 2015 while acryfentanyl and para-fluoroisobutyrfentanyl were controlled in 2016. By 2017, 15 fentanyl analogues and two precursor chemicals had been placed under international control and more than 20 individual substances had been detected in deaths. They have been routinely detected in drug markets in Europe and North America, and several more have been encountered in smaller, more isolated incidents, along with other non-morphine or fentanyl-related opioid receptor agonists. Up to now, two precursors and 25 fentanyl substances are regulated by China. In recent years, the number of people who have been addicted to fentanyl in the United States has risen sharply. Trump thus announced in October 2018 that the United States entered a "public health emergency." At the summit meeting of Chinese and American leaders held in December 2018, the leaders of two countries reached consensus on the control of fentanyl substances and this became a new bright spot in Sino-US cooperation.

Numerous analysis methods for fentanyl and its analogues have been published in the scientific literatures. Analysis technique such as liquid chromatography (HPLC) [5-9], gas chromatography/mass spectrometry (GC/MS) [10-18], liquid chromatography/high resolution mass spectrometry
(LC-HRMS) [19-20], liquid chromatography/tandem mass spectrometry (HPLC-MS/MS) [21-30] has been reported to apply in the precision determination and quantification of fentanyl and its analogues. GC/MS analysis method has been considered a gold standard for decades due to its combination of good selectivity and high specificity. HPLC-MS/MS analysis method provides excellent selectivity, as well as specificity and limits of detection that are usually better than those obtainable on GC/MS systems. On the other hand, surface enhanced Raman spectrometry has been applied for the rapid screening of fentanyl and its analogues [31-33]. However, they are all come with one critical limitation: they are "known unknown" method in which instrumental parameters must be specified for each compounds of interest, that is to say, standard substances of fentanyl and its analogues must be provided for the analysis. As a class of controlled substances, standard substances of fentanyl and its analogues are rather difficult to purchase. Therefore, not many types of fentanyl and its analogues have been preferred in all the existing literatures. It is mainly focused on fentanyl, alfentanil, remifentanil, sufentanil, carfentanyl and orfentanil. As to other fentanyl substances, they are rarely mentioned because of the difficulty to find standard or reference. An LC-MS/MS method has been established by Strayer et al. [30] to determine the contents of fentanyl and its analogues in blood, in which a total of 24 fentanyl and its analogues were detected. It is the most widely reported fentanyl species in the literature. However, it only covers 13 kinds of fentanyl substances controlled by China. At present, there are no reports in the literature on the determination of 27 fentanyl substances regulated in China.

In view of the difficulty in obtaining all controlled fentanyl standards, it is necessary to develop a screening method that does not require standards. Methods based upon full scan mass spectral data avoid the targeting limitations of the analytical technique mentioned above, but selectivity can suffer due to increased background, and specificity is hampered by the fact that most compounds of interest yield only a pseudomolecular ion under typical ESI and APCI conditions. Acquiring MS data with high spectral resolution and high mass accuracy can counter both these problems: high resolution can restore selectivity by permitting a given analyte signal to be extracted from a near-isobaric background signal, while mass accuracy improves specificity by restricting the number of possible chemical formulae that might give signals indistinguishable from the analyte of interest. Some chromatographic separation must still be retained in order to distinguishing true isobaric compounds. The increased availability of robust and easy to use high resolution mass spectrometers over the last few years has finally allowed people to begin realizing the potential of full scan MS methods in practice. This can be seen in the increasing number of broad-spectrum drug screening methods employing either time-of-flight or Orbitrap technology that have appeared within the last few years [34-40]. In this paper we present an ultra-high performance liquid chromatography/Orbitrap high resolution mass spectrometry (UPLC/Orbitrap HRMS) technique to simultaneously screen 
for the presence of 27 compounds, including two precursors and 25 fentanyl substances.

\section{Experimental}

Formic acid (purity $>98.0 \%$ ) and ammonium formate (purity $>97.0 \%$ ) were purchased from CNW Technologist Co, Germany. Acetonitrile and methanol (both HPLC grade) were purchased from Fisher Scientific, Pittsburgh, Pennsylvania,
USA. Fentanyl standard (purity 98.5\%) was purchased from Alta Scientific Co. Ltd, USA. De-ionized water (18+ mega-ohm grade) was obtained from an inhouse Millipore purification system. An UPLC analytical column (Accucore Phenylhexyl $\left.\mathrm{C}_{18}, 100 \mathrm{~mm} \times 2.1 \mathrm{~mm}, 1.6 \mu \mathrm{m}\right)$ was purchased from ThermoFisher Scientific, Bremen, Germany. Information of 27 fentanyl substances studied in this paper was listed in Table 1 .

Table 1. Information of 27 fentanyl substances.

\begin{tabular}{|c|c|c|c|c|}
\hline No. & Name & IUPAC Name & Formula & CAS No \\
\hline $1 \#$ & acetyl-alpha-methylfentanyl & $N$-Phenyl- $N$-[1-(1-phenylpropan-2-yl) piperidin-4-yl]acetamide & $\mathrm{C}_{22} \mathrm{H}_{28} \mathrm{~N}_{2} \mathrm{O}$ & $101860-00-8$ \\
\hline 2\# & Fentanyl & $\begin{array}{l}N \text {-Phenyl- } N \text {-[1-(2-phenylethyl)piperidin -4-yl]propanamide } \\
N \text {-\{1-[2-(4-Ethyl-5-oxo-4,5-dihydro- }\end{array}$ & $\mathrm{C}_{22} \mathrm{H}_{28} \mathrm{~N}_{2} \mathrm{O}$ & $437-38-7$ \\
\hline 4\# & alpha-methyl fentanyl & $N$-Phenyl- $N$-[1-(1-phenyl-2-propanyl) -4-piperidinyl]propanamide & $\mathrm{C}_{23} \mathrm{H}_{30} \mathrm{~N}_{2} \mathrm{O}$ & $79704-88-4$ \\
\hline $5 \#$ & 3-Methylfentanyl & $N$-[3-Methyl-1-(2-phenylethyl)-4-piperidinyl] $-N$-phenylpropanamide & $\mathrm{C}_{23} \mathrm{H}_{30} \mathrm{~N}_{2} \mathrm{O}$ & $42045-86-3$ \\
\hline 7\# & Isobutyryl fentanyl & 2-Methyl- $N$-phenyl- $N$-[1-(2-phenylethyl) -4-piperidinyl]propanamide & $\mathrm{C}_{23} \mathrm{H}_{30} \mathrm{~N}_{2} \mathrm{O}$ & $119618-70-1$ \\
\hline $8 \#$ & alpha-Methyl thiofentanyl & $N$-Phenyl- $N$-\{1-[1-(2-thienyl)-2-propanyl] -4-piperidinyl $\}$ propanamide & $\mathrm{C}_{21} \mathrm{H}_{28} \mathrm{~N}_{2} \mathrm{OS}$ & $103963-66-2$ \\
\hline 9\# & 3-Methyl thiofentanyl & cis- $N$-[3-Methyl-1-(2-thienyl)ethyl]-4- piperidinyl]- $N$-phenyl-propanamide & $\mathrm{C}_{21} \mathrm{H}_{28} \mathrm{~N}_{2} \mathrm{OS}$ & $86052-04-2$ \\
\hline 10\# & Beta-Hydroxy fentanyl & $N$-[1-(2-Hydroxy-2-phenylethyl)-4- piperidinyl]- $N$-phenylpropanamide & $\mathrm{C}_{22} \mathrm{H}_{28} \mathrm{~N}_{2} \mathrm{O}_{2}$ & $78995-10-5$ \\
\hline $11 \#$ & Beta-hydroxy-3-methylfentanyl & $\begin{array}{l}N \text {-[1-(2-Hydroxy-2-phenylethyl)-3-methylpiperidin } \\
\text {-4-yl]- } N \text {-phenylpropanamide }\end{array}$ & $\mathrm{C}_{23} \mathrm{H}_{30} \mathrm{~N}_{2} \mathrm{O}_{2}$ & 78995-14-9 \\
\hline $12 \#$ & para-Fluorofentanyl & $N$-(4-Fluorophenyl)- $N$-[1-(2-phenylethyl)-4- piperidinyl]propanamide & $\mathrm{C}_{22} \mathrm{H}_{27} \mathrm{FN}_{2} \mathrm{O}$ & $90736-23-5$ \\
\hline $14 \#$ & Sufentanil & $\begin{array}{l}N \text {-\{4-(Methoxymethyl)-1-[2-(2-thienyl)ethyl]-4- } \\
\text { piperidinyl }\}-N \text {-phenylpropanamide }\end{array}$ & $\mathrm{C}_{22} \mathrm{H}_{30} \mathrm{~N}_{2} \mathrm{O}_{2} \mathrm{~S}$ & $56030-54-7$ \\
\hline $15 \#$ & Thiofentanyl & $N$-Phenyl- $N$ - $\{1-[2-(2$-thienyl)ethyl] -4-piperidinyl $\}$ propanamide & $\mathrm{C}_{20} \mathrm{H}_{26} \mathrm{~N}_{2} \mathrm{OS}$ & $1165-22-6$ \\
\hline $16 \#$ & Acetyl fentanyl & N-Phenyl-N-[1-(2-phenylethyl) -4-piperidinyl]acetamide & $\mathrm{C}_{21} \mathrm{H}_{26} \mathrm{~N}_{2} \mathrm{O}$ & $3258-84-2$ \\
\hline $17 \#$ & beta-Hydroxythiofentanyl & $N$-\{1-[2-Hydroxy-2-(2-thienyl)ethyl] -4-piperidinyl $\}-N$-phenylpropanamide & $\mathrm{C}_{20} \mathrm{H}_{26} \mathrm{~N}_{2} \mathrm{O}_{2} \mathrm{~S}$ & $1474-34-6$ \\
\hline $18 \#$ & para-Fluorobutyryl fentanyl & $N$-(4-Fluorophenyl)- $N$-[1-(2-phenylethyl) -4-piperidinyl]butanamide & $\mathrm{C}_{23} \mathrm{H}_{29} \mathrm{FN}_{2} \mathrm{O}$ & 244195-31-1 \\
\hline 19\# & para-Fluoro-isobutyrylfentanyl & $N$-(4-Fluorophenyl)-2-methyl- $N$-[1- (2-phenylethyl)piperidin-4-yl]propanamide & $\mathrm{C}_{23} \mathrm{H}_{29} \mathrm{FN}_{2} \mathrm{O}$ & $244195-32-2$ \\
\hline $20 \#$ & Ocfentanil & $N$-(2-Fluorophenyl)-2-methoxy- $N$-[1- (2-phenylethyl)-4-piperidinyl]acetamide & $\mathrm{C}_{22} \mathrm{H}_{27} \mathrm{FN}_{2} \mathrm{O}_{2}$ & $101343-69-5$ \\
\hline 21\# & Carfentanil & $\begin{array}{l}\text { Methyl 1-(2-phenylethyl)-4-[phenyl (propionyl) } \\
\text { amino]-4-piperidinecarboxylate }\end{array}$ & $\mathrm{C}_{24} \mathrm{H}_{30} \mathrm{~N}_{2} \mathrm{O}_{3}$ & $59708-52-0$ \\
\hline 22\# & Furanyl fentanyl & $N$-Phenyl- $N$-[1-(2-phenylethyl)-4- piperidinyl]-2-furamide & $\mathrm{C}_{24} \mathrm{H}_{26} \mathrm{~N}_{2} \mathrm{O}_{2}$ & $101345-66-8$ \\
\hline 23\# & Acryl fentanyl & $N$-Phenyl- $N$-[1-(2-phenylethyl)-4- piperidinyl]acrylamide & $\mathrm{C}_{22} \mathrm{H}_{26} \mathrm{~N}_{2} \mathrm{O}$ & $82003-75-6$ \\
\hline 24\# & Valeryl fentanyl & $N$-Phenyl- $N$-[1-(2-phenylethyl)- 4-piperidinyl]pentanamide & $\mathrm{C}_{24} \mathrm{H}_{32} \mathrm{~N}_{2} \mathrm{O}$ & $122882-90-0$ \\
\hline $25 \#$ & tetrahydrofuranyl fentanyl & $\mathrm{N}-(1$-phenethylpiperidin-4-yl)-N- phenyltetrahydrofuran-2-carboxamide & $\mathrm{C}_{24} \mathrm{H}_{30} \mathrm{~N}_{2} \mathrm{O}_{2}$ & $2142571-01-3$ \\
\hline 26\# & NPP & N-phenethyl-4-piperidone & $\mathrm{C}_{13} \mathrm{H}_{17} \mathrm{NO}$ & $39742-60-4$ \\
\hline
\end{tabular}

Accurately weigh $10 \mathrm{mg}$ of the sample, dissolve it with $10 \mathrm{~mL}$ of acetonitrile, then dilute to the appropriate concentration, followed by UPLC/Orbitrap HRMS analysis.

Analysis was carried out with a Dionex Ultimate 3000-Q Exactive ultra-high performance liquid chromatography/ Orbitrap high resolution mass spectrometry supplied by ThermoFisher Scientific, Bremen, Germany.

Chromatographic separation was performed on an Accucore Phenylhexyl column $(100 \mathrm{~mm} \times 2.1 \mathrm{~mm} \times 2.6 \mu \mathrm{m})$ supplied by ThermoFisher Scientific, Bremen, Germany. A binary gradient separation was used with mobile phase of aqueous $2 \mathrm{mmol} / 1$ ammonium formate with $0.1 \%$ formic acid (mobile phase A), and 49.5\% methanol/49.5\%acetonitrile $11.0 \%$ water, containing $0.1 \%$ formic acid and $2 \mathrm{mmol} / 1$ ammonium formate (mobile phase B). The initial gradient start at $99 \%$ mobile phase A and $1 \%$ mobile phase $\mathrm{B}$, and maintained for 1.0 minute, followed by a gradual ramp to $1 \%$ mobile phase A and $99 \%$ mobile phase $\mathrm{B}$ at 10.0 minute. This mobile phase maintained to 11.5 minute, and then re-equilibrated back to the initial conditions for the next injection with a total runtime of 15.5 minutes. The autosampler needle and loop, and injection valve were washed with $800 \mu \mathrm{l}$ of $45 / 40 / 10 / 5$ methanol/acetonitrile/water /isopropanol (strong wash), followed by $1000 \mu \mathrm{l}$ of $90 / 10$ water/acetonitrile (weak wash). An injection volume of $1 \mu \mathrm{l}$, a flow rate of $0.5 \mu \mathrm{l} / \mathrm{min}$, an autosampler compartment temperature of $7^{\circ} \mathrm{C}$ and a column temperature of $40^{\circ} \mathrm{C}$ were used for the entire study.

The mass spectrometry analysis was performed using electrospray ionization in the positive mode with a HESI II probe at an inlet capillary temperature of $320^{\circ} \mathrm{C}$ and spray voltage of $3200 \mathrm{~V}$. Sheath and auxiliary gas flows of 50 and 10 arbitrary unites respectively were used. The temperature of auxiliary gas was $400^{\circ} \mathrm{C}$. Data acquisition on the Q-Exactive 
was performed with Xcalibur version 2.2, SP1.48, and tune page version 2.2, SP1. Data were collected with a resolution of 35000 in the full mass scan mode and 17500 in ddMS $^{2}$ mode over a scan range from $\mathrm{m} / \mathrm{z} 50$ to $\mathrm{m} / \mathrm{z} 750$. Data were collected in profile mode with maximum IT time of $50 \mathrm{~ms}$ and automatic gain control setting of 1e6. Identification of the target compounds was performed using the retention time and the accurate mass of the quasi-molecular ion. Confirmation was carried out by secondary ion segments with a mass tolerance of $5 \mathrm{ppm}$.

\section{Results and Discussion}

\subsection{Establishment of the Screening List}

Only one peak existed in the full scan mass spectra of 27 fentanyl substances in $\mathrm{ESI}^{+}$mode, and this peak was the quasi-molecular ion of 27 fentanyl substances. It was broken under a certain collision voltage to produce secondary ion segments. The fracture mechanism of 27 fentanyl substance was illustrated taking fentanyl as an example. The $\mathrm{C}-\mathrm{N}$ bond of the N-phenylamide moiety in quansi-molecular ion $[\mathrm{M}+\mathrm{H}]^{+}$ of fentanyl is cleaved, and a propionaldehyde group is removed to form ion segment $\mathrm{A}$ while the $\mathrm{C}-\mathrm{N}$ bond and $\mathrm{C}-\mathrm{C}$ bond in the piperidine ring are broken to generate ion segment $\mathrm{F}$ and ion segment $\mathrm{G}$. Ion segment $\mathrm{A}$ can be further broken to form ion segment $\mathrm{B}$ and ion segment $\mathrm{C}$. Quansi-molecular ion $[\mathrm{M}+\mathrm{H}]^{+}$can also be broken to form ion segment D. Piperidine ring and N-phenylamide substituent in quansi-molecular ion can also be cleaved to form ion segment E. Figure 1 shows the possible cleavage pathway for fentanyl. The fracture mechanism of other fentanyl species is similar to that of fentanyl except for precursor NPP (26\#) and 4-ANPP (27\#). No ion segment A appears in secondary ion segments of 4-ANPP. Similarly, no ion segment A, C and G appear in secondary ion segments of NPP. On the other hand, the piperidine ring in NPP is broken and a carbonyl group is lost, ring closure occurs to form ion segment E. Information of the quansi-molecular ion and secondary ion fragments of the 27 target analytes are shown in Table 2. The serial number of each target analyte in Table 2 is the same as Table 1.

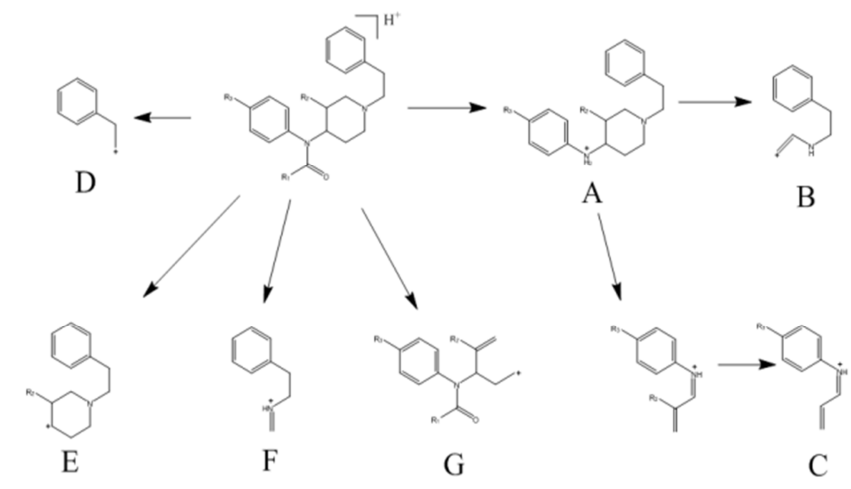

Figure 1. Proposed fragmentation pathways of product ion for fentabyl in $E S I^{+}$mode.

Table 2. Quasi-molecular ion and secondary ion fragment of 27 fentanyl substances $/ \mathrm{m} / \mathrm{z}$.

\begin{tabular}{|c|c|c|c|c|c|c|c|c|c|}
\hline No. & & {$[\mathbf{M}+\mathbf{H}]$} & Segment A & Segment B & Segment C & Segment D & Segment E & Segment F & Segment G \\
\hline \multirow[t]{2}{*}{$1 \#$} & formula & $\mathrm{C}_{22} \mathrm{H}_{29} \mathrm{~N}_{2} \mathrm{O}$ & $\mathrm{C}_{20} \mathrm{H}_{27} \mathrm{~N}_{2}$ & $\mathrm{C}_{11} \mathrm{H}_{14} \mathrm{~N}$ & $\mathrm{C}_{9} \mathrm{H}_{10} \mathrm{~N}$ & $\mathrm{C}_{9} \mathrm{H}_{11}$ & $\mathrm{C}_{14} \mathrm{H}_{20} \mathrm{~N}$ & $\mathrm{C}_{10} \mathrm{H}_{14} \mathrm{~N}$ & $\mathrm{C}_{13} \mathrm{H}_{16} \mathrm{NO}$ \\
\hline & accurate mass & 337.22744 & 295.21168 & 160.11208 & 132.08078 & 119.08553 & 202.15903 & 148.11208 & 202.12264 \\
\hline \multirow[t]{2}{*}{$2 \#$} & formula & $\mathrm{C}_{22} \mathrm{H}_{29} \mathrm{~N}_{2} \mathrm{O}$ & $\mathrm{C}_{19} \mathrm{H}_{25} \mathrm{~N}_{2}$ & $\mathrm{C}_{10} \mathrm{H}_{12} \mathrm{~N}$ & $\mathrm{C}_{9} \mathrm{H}_{10} \mathrm{~N}$ & $\mathrm{C}_{8} \mathrm{H}_{9}$ & $\mathrm{C}_{13} \mathrm{H}_{18} \mathrm{~N}$ & $\mathrm{C}_{9} \mathrm{H}_{12} \mathrm{~N}$ & $\mathrm{C}_{14} \mathrm{H}_{18} \mathrm{NO}$ \\
\hline & accurate mass & 337.22744 & 281.20123 & 146.09643 & 132.08078 & 105.06988 & 188.14338 & 134.09643 & 216.13829 \\
\hline \multirow[t]{2}{*}{$3 \#$} & formula & $\mathrm{C}_{21} \mathrm{H}_{33} \mathrm{~N}_{6} \mathrm{O}_{3}$ & $\mathrm{C}_{18} \mathrm{H}_{29} \mathrm{~N}_{6} \mathrm{O}_{2}$ & $\mathrm{C}_{7} \mathrm{H}_{12} \mathrm{~N}_{5} \mathrm{O}$ & $\mathrm{C}_{11} \mathrm{H}_{14} \mathrm{NO}$ & $\mathrm{C}_{5} \mathrm{H}_{9} \mathrm{~N}_{4} \mathrm{O}$ & $\mathrm{C}_{12} \mathrm{H}_{22} \mathrm{~N}_{5} \mathrm{O}_{2}$ & $\mathrm{C}_{6} \mathrm{H}_{12} \mathrm{~N}_{5} \mathrm{O}$ & $\mathrm{C}_{16} \mathrm{H}_{22} \mathrm{NO}_{2}$ \\
\hline & accurate mass & 417.26087 & 361.23465 & 182.10364 & 176.10699 & 141.07709 & 268.17680 & 170.10364 & 260.16451 \\
\hline \multirow[t]{2}{*}{ 4\# } & formula & $\mathrm{C}_{23} \mathrm{H}_{31} \mathrm{~N}_{2} \mathrm{O}$ & $\mathrm{C}_{20} \mathrm{H}_{27} \mathrm{~N}_{2}$ & $\mathrm{C}_{11} \mathrm{H}_{14} \mathrm{~N}$ & $\mathrm{C}_{9} \mathrm{H}_{10} \mathrm{~N}$ & $\mathrm{C}_{9} \mathrm{H}_{11}$ & $\mathrm{C}_{14} \mathrm{H}_{20} \mathrm{~N}$ & $\mathrm{C}_{10} \mathrm{H}_{14} \mathrm{~N}$ & $\mathrm{C}_{14} \mathrm{H}_{18} \mathrm{NO}$ \\
\hline & accurate mass & 351.24309 & 295.21688 & 160.11208 & 132.08078 & 119.08553 & 202.15903 & 148.11208 & 216.13829 \\
\hline \multirow[t]{2}{*}{$5 \#$} & formula & $\mathrm{C}_{23} \mathrm{H}_{31} \mathrm{~N}_{2} \mathrm{O}$ & $\mathrm{C}_{20} \mathrm{H}_{27} \mathrm{~N}_{2}$ & $\mathrm{C}_{10} \mathrm{H}_{12} \mathrm{~N}$ & $\mathrm{C}_{9} \mathrm{H}_{10} \mathrm{~N}$ & $\mathrm{C}_{8} \mathrm{H}_{9}$ & $\mathrm{C}_{14} \mathrm{H}_{20} \mathrm{~N}$ & $\mathrm{C}_{9} \mathrm{H}_{12} \mathrm{~N}$ & $\mathrm{C}_{15} \mathrm{H}_{20} \mathrm{NO}$ \\
\hline & accurate mass & 351.24309 & 295.21168 & 146.09643 & 132.08078 & 105.06988 & 202.15903 & 134.09643 & 230.15394 \\
\hline \multirow[t]{2}{*}{$6 \#$} & formula & $\mathrm{C}_{23} \mathrm{H}_{31} \mathrm{~N}_{2} \mathrm{O}$ & $\mathrm{C}_{19} \mathrm{H}_{25} \mathrm{~N}_{2}$ & $\mathrm{C}_{10} \mathrm{H}_{12} \mathrm{~N}$ & $\mathrm{C}_{9} \mathrm{H}_{10} \mathrm{~N}$ & $\mathrm{C}_{8} \mathrm{H}_{9}$ & $\mathrm{C}_{13} \mathrm{H}_{18} \mathrm{~N}$ & $\mathrm{C}_{9} \mathrm{H}_{12} \mathrm{~N}$ & $\mathrm{C}_{15} \mathrm{H}_{20} \mathrm{NO}$ \\
\hline & accurate mass & 351.24309 & 281.20123 & 146.09643 & 132.08078 & 105.06988 & 188.14338 & 134.09643 & 230.15394 \\
\hline \multirow[t]{2}{*}{ 7\# } & formula & $\mathrm{C}_{23} \mathrm{H}_{31} \mathrm{~N}_{2} \mathrm{O}$ & $\mathrm{C}_{19} \mathrm{H}_{25} \mathrm{~N}_{2}$ & $\mathrm{C}_{10} \mathrm{H}_{12} \mathrm{~N}$ & $\mathrm{C}_{9} \mathrm{H}_{10} \mathrm{~N}$ & $\mathrm{C}_{8} \mathrm{H}_{9}$ & $\mathrm{C}_{13} \mathrm{H}_{18} \mathrm{~N}$ & $\mathrm{C}_{9} \mathrm{H}_{12} \mathrm{~N}$ & $\mathrm{C}_{15} \mathrm{H}_{20} \mathrm{NO}$ \\
\hline & accurate mass & 351.24309 & 281.20123 & 146.09643 & 132.08078 & 105.06988 & 188.14338 & 134.09643 & 230.15394 \\
\hline \multirow[t]{2}{*}{$8 \#$} & formula & $\mathrm{C}_{21} \mathrm{H}_{29} \mathrm{~N}_{2} \mathrm{OS}$ & $\mathrm{C}_{18} \mathrm{H}_{25} \mathrm{~N}_{2} \mathrm{~S}$ & $\mathrm{C}_{9} \mathrm{H}_{12} \mathrm{NS}$ & $\mathrm{C}_{9} \mathrm{H}_{10} \mathrm{~N}$ & $\mathrm{C}_{7} \mathrm{H}_{9} \mathrm{~S}$ & $\mathrm{C}_{12} \mathrm{H}_{18} \mathrm{NS}$ & $\mathrm{C}_{8} \mathrm{H}_{12} \mathrm{NS}$ & $\mathrm{C}_{14} \mathrm{H}_{18} \mathrm{NO}$ \\
\hline & accurate mass & 357.19951 & 301.17330 & 166.06850 & 132.08078 & 125.04195 & 208.11545 & 154.06850 & 216.13829 \\
\hline \multirow[t]{2}{*}{ 9\# } & formula & $\mathrm{C}_{21} \mathrm{H}_{29} \mathrm{~N}_{2} \mathrm{OS}$ & $\mathrm{C}_{18} \mathrm{H}_{25} \mathrm{~N}_{2} \mathrm{~S}$ & $\mathrm{C}_{8} \mathrm{H}_{10} \mathrm{NS}$ & $\mathrm{C}_{9} \mathrm{H}_{10} \mathrm{~N}$ & $\mathrm{C}_{6} \mathrm{H}_{7} \mathrm{~S}$ & $\mathrm{C}_{12} \mathrm{H}_{18} \mathrm{NS}$ & $\mathrm{C}_{7} \mathrm{H}_{10} \mathrm{NS}$ & $\mathrm{C}_{15} \mathrm{H}_{20} \mathrm{NO}$ \\
\hline & accurate mass & 357.19951 & 301.17330 & 152.05285 & 132.08078 & 111.02630 & 208.11545 & 140.05285 & 230.15394 \\
\hline \multirow[t]{2}{*}{$10 \#$} & formula & $\mathrm{C}_{22} \mathrm{H}_{29} \mathrm{~N}_{2} \mathrm{O}_{2}$ & $\mathrm{C}_{19} \mathrm{H}_{25} \mathrm{~N}_{2} \mathrm{O}$ & $\mathrm{C}_{10} \mathrm{H}_{12} \mathrm{NO}$ & $\mathrm{C}_{9} \mathrm{H}_{10} \mathrm{~N}$ & $\mathrm{C}_{8} \mathrm{H}_{9} \mathrm{O}$ & $\mathrm{C}_{13} \mathrm{H}_{18} \mathrm{NO}$ & $\mathrm{C}_{9} \mathrm{H}_{12} \mathrm{NO}$ & $\mathrm{C}_{14} \mathrm{H}_{18} \mathrm{NO}$ \\
\hline & accurate mass & 353.22235 & 297.19614 & 162.09134 & 132.08078 & 121.06479 & 204.13829 & 150.09134 & 216.13829 \\
\hline \multirow[t]{2}{*}{$11 \#$} & formula & $\mathrm{C}_{23} \mathrm{H}_{31} \mathrm{~N}_{2} \mathrm{O}_{2}$ & $\mathrm{C}_{20} \mathrm{H}_{27} \mathrm{~N}_{2} \mathrm{O}$ & $\mathrm{C}_{10} \mathrm{H}_{12} \mathrm{NO}$ & $\mathrm{C}_{9} \mathrm{H}_{10} \mathrm{~N}$ & $\mathrm{C}_{8} \mathrm{H}_{9} \mathrm{O}$ & $\mathrm{C}_{14} \mathrm{H}_{20} \mathrm{NO}$ & $\mathrm{C}_{9} \mathrm{H}_{12} \mathrm{NO}$ & $\mathrm{C}_{15} \mathrm{H}_{20} \mathrm{NO}$ \\
\hline & accurate mass & 367.23800 & 311.21179 & 162.09134 & 132.08078 & 121.06479 & 218.15394 & 150.09134 & 230.15394 \\
\hline \multirow[t]{2}{*}{$12 \#$} & formula & $\mathrm{C}_{22} \mathrm{H}_{28} \mathrm{~N}_{2} \mathrm{OF}$ & $\mathrm{C}_{19} \mathrm{H}_{24} \mathrm{~N}_{2} \mathrm{~F}$ & $\mathrm{C}_{10} \mathrm{H}_{12} \mathrm{~N}$ & $\mathrm{C}_{9} \mathrm{H}_{10} \mathrm{~N}$ & $\mathrm{C}_{8} \mathrm{H}_{9}$ & $\mathrm{C}_{13} \mathrm{H}_{18} \mathrm{~N}$ & $\mathrm{C}_{9} \mathrm{H}_{12} \mathrm{~N}$ & $\mathrm{C}_{14} \mathrm{H}_{17} \mathrm{NOF}$ \\
\hline & accurate mass & 355.21802 & 299.19180 & 146.09643 & 132.08078 & 105.06988 & 188.14338 & 134.09643 & 234.12887 \\
\hline \multirow[t]{2}{*}{$13 \#$} & formula & $\mathrm{C}_{20} \mathrm{H}_{29} \mathrm{~N}_{2} \mathrm{O}_{5}$ & $\mathrm{C}_{17} \mathrm{H}_{25} \mathrm{~N}_{2} \mathrm{O}_{4}$ & $\mathrm{C}_{6} \mathrm{H}_{10} \mathrm{NO}_{2}$ & $\mathrm{C}_{11} \mathrm{H}_{12} \mathrm{NO}_{2}$ & $\mathrm{C}_{4} \mathrm{H}_{7} \mathrm{O}_{2}$ & $\mathrm{C}_{11} \mathrm{H}_{18} \mathrm{NO}_{4}$ & $\mathrm{C}_{5} \mathrm{H}_{10} \mathrm{NO}_{2}$ & $\mathrm{C}_{16} \mathrm{H}_{20} \mathrm{NO}_{3}$ \\
\hline & accurate mass & 377.20710 & 321.18088 & 128.07061 & 190.08626 & 87.04406 & 228.12303 & 116.07061 & 274.14377 \\
\hline \multirow[t]{2}{*}{$14 \#$} & formula & $\mathrm{C}_{22} \mathrm{H}_{31} \mathrm{~N}_{2} \mathrm{O}_{2} \mathrm{~S}$ & $\mathrm{C}_{19} \mathrm{H}_{27} \mathrm{~N}_{2} \mathrm{OS}$ & $\mathrm{C}_{8} \mathrm{H}_{10} \mathrm{NS}$ & $\mathrm{C}_{11} \mathrm{H}_{14} \mathrm{NO}$ & $\mathrm{C}_{6} \mathrm{H}_{7} \mathrm{~S}$ & $\mathrm{C}_{13} \mathrm{H}_{20} \mathrm{NSO}$ & $\mathrm{C}_{7} \mathrm{H}_{10} \mathrm{NS}$ & $\mathrm{C}_{16} \mathrm{H}_{22} \mathrm{NO}_{2}$ \\
\hline & accurate mass & 387.21008 & 331.18386 & 152.05285 & 176.10699 & 111.02630 & 238.12601 & 140.05285 & 260.16451 \\
\hline \multirow[t]{2}{*}{$15 \#$} & formula & $\mathrm{C}_{20} \mathrm{H}_{27} \mathrm{~N}_{2} \mathrm{OS}$ & $\mathrm{C}_{17} \mathrm{H}_{23} \mathrm{~N}_{2} \mathrm{~S}$ & $\mathrm{C}_{8} \mathrm{H}_{10} \mathrm{NS}$ & $\mathrm{C}_{9} \mathrm{H}_{10} \mathrm{~N}$ & $\mathrm{C}_{6} \mathrm{H}_{7} \mathrm{~S}$ & $\mathrm{C}_{11} \mathrm{H}_{16} \mathrm{NS}$ & $\mathrm{C}_{7} \mathrm{H}_{10} \mathrm{NS}$ & $\mathrm{C}_{14} \mathrm{H}_{18} \mathrm{NO}$ \\
\hline & accurate mass & 343.18386 & 287.15765 & 152.05285 & 132.08078 & 111.02630 & 194.09980 & 140.05285 & 216.13829 \\
\hline \multirow[t]{2}{*}{$16 \#$} & formula & $\mathrm{C}_{21} \mathrm{H}_{27} \mathrm{~N}_{2} \mathrm{O}$ & $\mathrm{C}_{19} \mathrm{H}_{25} \mathrm{~N}_{2}$ & $\mathrm{C}_{10} \mathrm{H}_{12} \mathrm{~N}$ & $\mathrm{C}_{9} \mathrm{H}_{10} \mathrm{~N}$ & $\mathrm{C}_{8} \mathrm{H}_{9}$ & $\mathrm{C}_{13} \mathrm{H}_{18} \mathrm{~N}$ & $\mathrm{C}_{9} \mathrm{H}_{12} \mathrm{~N}$ & $\mathrm{C}_{13} \mathrm{H}_{16} \mathrm{NO}$ \\
\hline & accurate mass & 323.21179 & 281.20123 & 146.09643 & 132.08078 & 105.06988 & 188.14338 & 134.09643 & 202.12264 \\
\hline
\end{tabular}




\begin{tabular}{|c|c|c|c|c|c|c|c|c|c|}
\hline No. & & {$[\mathrm{M}+\mathrm{H}]$} & Segment A & Segment B & Segment C & Segment D & Segment E & Segment F & Segment G \\
\hline \multirow[t]{2}{*}{$17 \#$} & formula & $\mathrm{C}_{20} \mathrm{H}_{27} \mathrm{~N}_{2} \mathrm{O}_{2} \mathrm{~S}$ & $\mathrm{C}_{17} \mathrm{H}_{23} \mathrm{~N}_{2} \mathrm{SO}$ & $\mathrm{C}_{8} \mathrm{H}_{10} \mathrm{NSO}$ & $\mathrm{C}_{9} \mathrm{H}_{10} \mathrm{NO}$ & $\mathrm{C}_{6} \mathrm{H}_{7} \mathrm{SO}$ & $\mathrm{C}_{11} \mathrm{H}_{16} \mathrm{NSO}$ & $\mathrm{C}_{7} \mathrm{H}_{10} \mathrm{NSO}$ & $\mathrm{C}_{14} \mathrm{H}_{18} \mathrm{NO}$ \\
\hline & accurate mass & 359.17878 & 303.15256 & 182.05084 & 148.07569 & 127.02121 & 210.09471 & 156.04776 & 216.13829 \\
\hline \multirow[t]{2}{*}{$18 \#$} & formula & $\mathrm{C}_{23} \mathrm{H}_{30} \mathrm{~N}_{2} \mathrm{OF}$ & $\mathrm{C}_{19} \mathrm{H}_{24} \mathrm{~N}_{2} \mathrm{~F}$ & $\mathrm{C}_{10} \mathrm{H}_{12} \mathrm{~N}$ & $\mathrm{C}_{9} \mathrm{H}_{9} \mathrm{NF}$ & $\mathrm{C}_{8} \mathrm{H}_{9}$ & $\mathrm{C}_{13} \mathrm{H}_{18} \mathrm{~N}$ & $\mathrm{C}_{9} \mathrm{H}_{12} \mathrm{~N}$ & $\mathrm{C}_{15} \mathrm{H}_{19} \mathrm{NOF}$ \\
\hline & accurate mass & 369.23367 & 299.19180 & 146.09643 & 150.07135 & 105.06988 & 188.14338 & 134.09643 & 248.14452 \\
\hline \multirow{2}{*}{$19 \#$} & formula & $\mathrm{C}_{23} \mathrm{H}_{30} \mathrm{~N}_{2} \mathrm{OF}$ & $\mathrm{C}_{19} \mathrm{H}_{24} \mathrm{~N}_{2} \mathrm{~F}$ & $\mathrm{C}_{10} \mathrm{H}_{12} \mathrm{~N}$ & $\mathrm{C}_{9} \mathrm{H}_{9} \mathrm{NF}$ & $\mathrm{C}_{8} \mathrm{H}_{9}$ & $\mathrm{C}_{13} \mathrm{H}_{18} \mathrm{~N}$ & $\mathrm{C}_{9} \mathrm{H}_{12} \mathrm{~N}$ & $\mathrm{C}_{15} \mathrm{H}_{19} \mathrm{NOF}$ \\
\hline & accurate mass & 369.23367 & 299.19180 & 146.09643 & 150.07135 & 105.06988 & 188.14338 & 134.09643 & 248.14452 \\
\hline \multirow[t]{2}{*}{$20 \#$} & formula & $\mathrm{C}_{22} \mathrm{H}_{28} \mathrm{~N}_{2} \mathrm{O}_{2} \mathrm{~F}$ & $\mathrm{C}_{19} \mathrm{H}_{24} \mathrm{~N}_{2} \mathrm{~F}$ & $\mathrm{C}_{10} \mathrm{H}_{12} \mathrm{~N}$ & $\mathrm{C}_{9} \mathrm{H}_{9} \mathrm{NF}$ & $\mathrm{C}_{8} \mathrm{H}_{9}$ & $\mathrm{C}_{13} \mathrm{H}_{18} \mathrm{~N}$ & $\mathrm{C}_{9} \mathrm{H}_{12} \mathrm{~N}$ & $\mathrm{C}_{14} \mathrm{H}_{17} \mathrm{NO}_{2} \mathrm{~F}$ \\
\hline & accurate mass & 371.21293 & 299.19180 & 146.09643 & 150.07135 & 105.06988 & 188.14338 & 134.09643 & 250.12378 \\
\hline \multirow[t]{2}{*}{$21 \#$} & formula & $\mathrm{C}_{24} \mathrm{H}_{31} \mathrm{~N}_{2} \mathrm{O}_{3}$ & $\mathrm{C}_{21} \mathrm{H}_{27} \mathrm{~N}_{2} \mathrm{O}_{2}$ & $\mathrm{C}_{10} \mathrm{H}_{12} \mathrm{~N}$ & $\mathrm{C}_{11} \mathrm{H}_{12} \mathrm{NO}_{2}$ & $\mathrm{C}_{8} \mathrm{H}_{9}$ & $\mathrm{C}_{15} \mathrm{H}_{20} \mathrm{NO}_{2}$ & $\mathrm{C}_{9} \mathrm{H}_{12} \mathrm{~N}$ & $\mathrm{C}_{16} \mathrm{H}_{20} \mathrm{NO}_{3}$ \\
\hline & accurate mass & 395.23292 & 339.20670 & 146.09643 & 190.08626 & 105.06988 & 246.14886 & 134.09643 & 274.14377 \\
\hline \multirow[t]{2}{*}{$22 \#$} & formula & $\mathrm{C}_{24} \mathrm{H}_{27} \mathrm{~N}_{2} \mathrm{O}_{2}$ & $\mathrm{C}_{19} \mathrm{H}_{25} \mathrm{~N}_{2}$ & $\mathrm{C}_{10} \mathrm{H}_{12} \mathrm{~N}$ & $\mathrm{C}_{9} \mathrm{H}_{10} \mathrm{~N}$ & $\mathrm{C}_{8} \mathrm{H}_{9}$ & $\mathrm{C}_{13} \mathrm{H}_{18} \mathrm{~N}$ & $\mathrm{C}_{9} \mathrm{H}_{12} \mathrm{~N}$ & $\mathrm{C}_{16} \mathrm{H}_{16} \mathrm{NO}_{2}$ \\
\hline & accurate mass & 375.20670 & 281.20123 & 146.09643 & 132.08078 & 105.06988 & 188.14338 & 134.09643 & 254.11756 \\
\hline \multirow[t]{2}{*}{$23 \#$} & formula & $\mathrm{C}_{22} \mathrm{H}_{27} \mathrm{~N}_{2} \mathrm{O}$ & $\mathrm{C}_{19} \mathrm{H}_{25} \mathrm{~N}_{2}$ & $\mathrm{C}_{10} \mathrm{H}_{12} \mathrm{~N}$ & $\mathrm{C}_{9} \mathrm{H}_{10} \mathrm{~N}$ & $\mathrm{C}_{8} \mathrm{H}_{9}$ & $\mathrm{C}_{13} \mathrm{H}_{18} \mathrm{~N}$ & $\mathrm{C}_{9} \mathrm{H}_{12} \mathrm{~N}$ & $\mathrm{C}_{14} \mathrm{H}_{16} \mathrm{NO}$ \\
\hline & accurate mass & 335.21179 & 281.20123 & 146.09643 & 132.08078 & 105.06988 & 188.14338 & 134.09643 & 2264 \\
\hline \multirow[t]{2}{*}{$24 \#$} & formula & $\mathrm{C}_{24} \mathrm{H}_{33} \mathrm{~N}_{2} \mathrm{O}$ & $\mathrm{C}_{19} \mathrm{H}_{25} \mathrm{~N}_{2}$ & $\mathrm{C}_{10} \mathrm{H}_{12} \mathrm{~N}$ & $\mathrm{C}_{9} \mathrm{H}_{10} \mathrm{~N}$ & $\mathrm{C}_{8} \mathrm{H}_{9}$ & $\mathrm{C}_{13} \mathrm{H}_{18} \mathrm{~N}$ & $\mathrm{C}_{9} \mathrm{H}_{12} \mathrm{~N}$ & $\mathrm{C}_{16} \mathrm{H}_{22} \mathrm{NO}$ \\
\hline & accurate mass & 365.25874 & 281.20123 & 146.09643 & 132.08078 & 105.06988 & 188.14338 & 134.09643 & 244.16959 \\
\hline \multirow[t]{2}{*}{$25 \#$} & formula & $\mathrm{C}_{24} \mathrm{H}_{31} \mathrm{~N}_{2} \mathrm{O}_{2}$ & $\mathrm{C}_{19} \mathrm{H}_{25} \mathrm{~N}_{2}$ & $\mathrm{C}_{10} \mathrm{H}_{12} \mathrm{~N}$ & $\mathrm{C}_{9} \mathrm{H}_{10} \mathrm{~N}$ & $\mathrm{C}_{8} \mathrm{H}_{9}$ & $\mathrm{C}_{13} \mathrm{H}_{18} \mathrm{~N}$ & $\mathrm{C}_{9} \mathrm{H}_{12} \mathrm{~N}$ & $\mathrm{C}_{16} \mathrm{H}_{20} \mathrm{NO}_{2}$ \\
\hline & accurate mass & 379.23800 & 281.20123 & 146.09643 & 132.08078 & 105.06988 & 188.14338 & 134.09643 & 258.14886 \\
\hline \multirow[t]{2}{*}{$26 \#$} & formula & $\mathrm{C}_{13} \mathrm{H}_{18} \mathrm{NO}$ & - & $\mathrm{C}_{10} \mathrm{H}_{12} \mathrm{~N}$ & - & $\mathrm{C}_{8} \mathrm{H}_{9}$ & $\mathrm{C}_{12} \mathrm{H}_{18} \mathrm{~N}$ & $\mathrm{C}_{9} \mathrm{H}_{12} \mathrm{~N}$ & - \\
\hline & accurate mass & 204.13829 & - & 146.09643 & - & 105.06988 & 176.14338 & 134.09643 & - \\
\hline \multirow[t]{2}{*}{$27 \#$} & formula & $\mathrm{C}_{19} \mathrm{H}_{25} \mathrm{~N}_{2}$ & - & $\mathrm{C}_{10} \mathrm{H}_{12} \mathrm{~N}$ & $\mathrm{C}_{9} \mathrm{H}_{10} \mathrm{~N}$ & $\mathrm{C}_{8} \mathrm{H}_{9}$ & $\mathrm{C}_{13} \mathrm{H}_{18} \mathrm{~N}$ & $\mathrm{C}_{9} \mathrm{H}_{12} \mathrm{~N}$ & $\mathrm{C}_{11} \mathrm{H}_{14} \mathrm{~N}$ \\
\hline & accurate mass & 281.20123 & - & 146.09643 & 132.08078 & 105.06988 & 188.14338 & 134.09643 & 160.11208 \\
\hline
\end{tabular}

-: segment did not appear

A database was created using the accurate mass and formula of quasi-molecular ion and secondary ion segments listed in Table 2, then this database was used to establish a screening list utilizing TraceFinder software, in which rapid screening of the analytes was performed using the accurate mass of the quansi-molecular ion and confirmation was performed utilizing the accurate mass and formula of secondary ion segments while the accurate mass deviation less than $5 \times 10^{-6}$. At least 4.0 identification points are required to confirm an analyte using secondary mass spectrometry according to EU Directive 2002/657/EC while a primary ion provided 2.0 identification points and a secondary ion segment provided 2.5 identification points. In order to ensure the reliability of the confirmation results, at least 4 secondary ion segments were provided for each target analyte, and the identification point were all larger than 12.0, far exceeding the identification requirements of 2002/657/EC.

\subsection{Rapid Screening and Conformation of Real Samples}

The proposed method was used for the rapid screening of the target compounds in nine white powder samples transferred from the customs anti-smuggling department and the results showed that both fentanyl and precursor 4-ANPP existed simultaneously in sample $1 \#$ and sample $2 \#$ while fentanyl, acetylfentanyl and precursor 4-ANPP were detected simultaneously in other seven samples. Figure 2(a) and Figure 2(b) were UPLC/Orbitrap HRMS extracted ion chromatograms of samples $1 \#$ and $3 \#$, respectively. The peaks appearing at $5.20 \mathrm{~min}$ and $5.42 \mathrm{~min}$ in Figure 2(a) were 4-ANPP and fentanyl, respectively. The peaks appearing at $4.93 \mathrm{~min}, 5.20 \mathrm{~min}$ and $5.42 \mathrm{~min}$ in Figure 2(b) were acetylfentanyl, 4-ANPP and fentanyl, respectively.

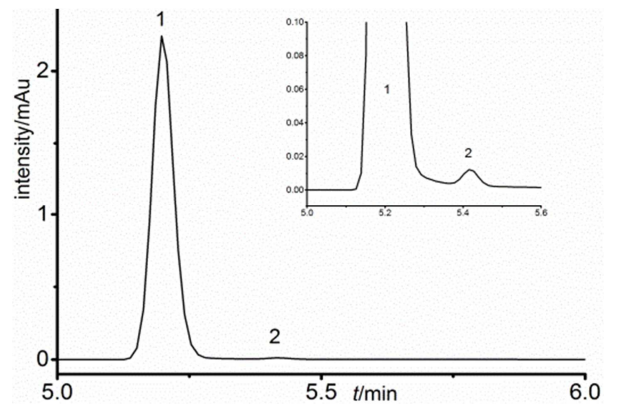

(a)

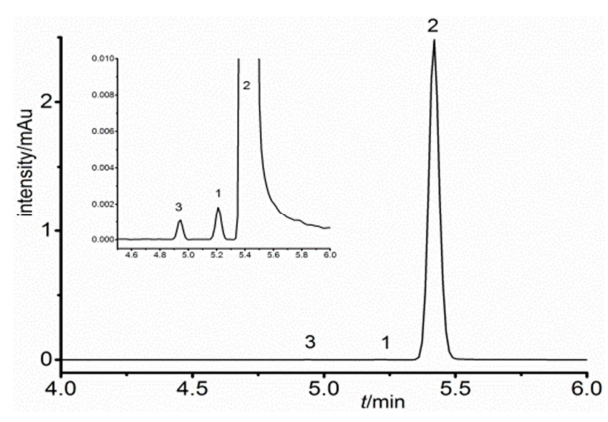

(b)

Figure 2. Extracted ion chromatograms of real samples, (a) sample 1\#; (b) sample 3\#, 1:4-ANPP; 2: fentanyl; 3: acetylfentanyl.

In $\mathrm{ESI}^{+}$mode, the formula of the quasi-molecular ion $[\mathrm{M}+\mathrm{H}]^{+}$of 4-ANPP, fentanyl and acetylfentanyl are $\mathrm{C}_{19} \mathrm{H}_{25} \mathrm{~N}_{2}$, $\mathrm{C}_{22} \mathrm{H}_{29} \mathrm{~N}_{2} \mathrm{O}, \mathrm{C}_{21} \mathrm{H}_{27} \mathrm{~N}_{2} \mathrm{O}$, respectively. The information of secondary ion segments that may be generated after fracture was shown in Table 2 but some of the secondary ion segments may disappear completely. Secondary mass spectrometry analysis was performed for sample $3 \#$ in which 4-ANPP, fentanyl and acetylfentanyl were detected simultaneously as shown in Figure 2(b). The results were shown in Figure 3, all 
other secondary ion segments were detected except for ion segment $A$ and $G$ in precursor 4-ANPP and ion segment $A$ in acetylfentanyl. The theorectical and measured values of accurate mass of quasi-molecular ion and secondary ion segments of 4-ANPP, fentanyl and acetylfentanyl detected in the 3\# sample were listed in Table 3. The deviation from the corresponding theoretical values was calculated and also shown in Table 3. The deviation changed in the range from $-4.13 \times 10^{-6}$ to $+0.48 \times 10^{-6}$, and all less than $\pm 5 \times 10^{-6}$, meeting the requirements of the high-resolution mass spectrometric identification of the European Union Regulation SANCO/12571/2013. For comparison, fentanyl standard was also analyzed and its secondary ion segments were also listed in Table 3. The accurate mass of ion segment $C$ and $D$ is the same in sample and fentanyl standard. The deviation of accurate mass of quansi-molecular ion and ion segment A, B, $\mathrm{E}, \mathrm{F}$ and $\mathrm{G}$ in sample and fentanyl standard was all less than $\pm 5 \times 10^{-6}$. That is to say, fentanyl was really detected in the $3 \#$ sample. European Directive 2002/657/EC stipulates that at least 4.0 identification points are required for confirmation using secondary mass spectrometry. In this paper, one primary parent ion and five to seven secondary ion segments were used for confirmation, and the identification point was high as 14.5 to 19.5 . Therefore, 4-ANPP, fentanyl and acetylfentanyl were confirmed to exist in $3 \#$ sample.

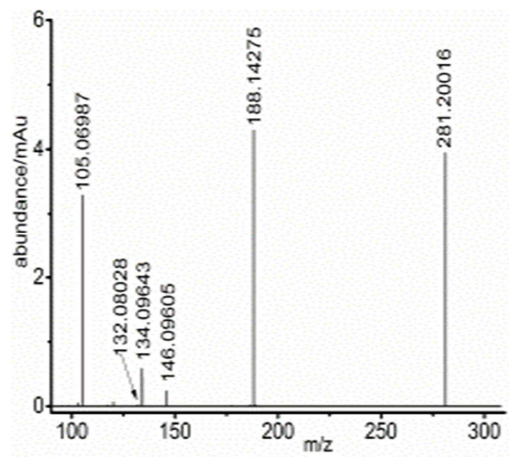

(a)

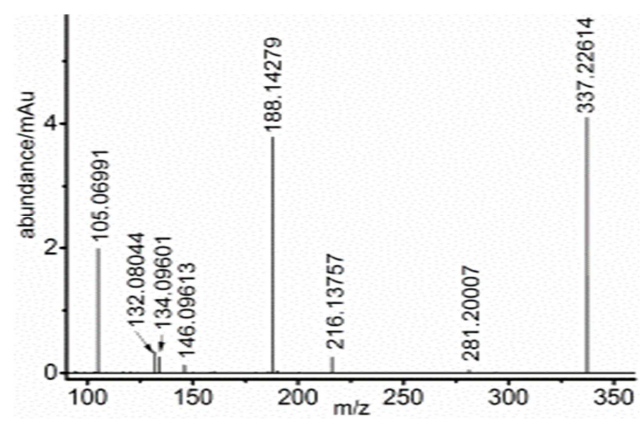

(b)

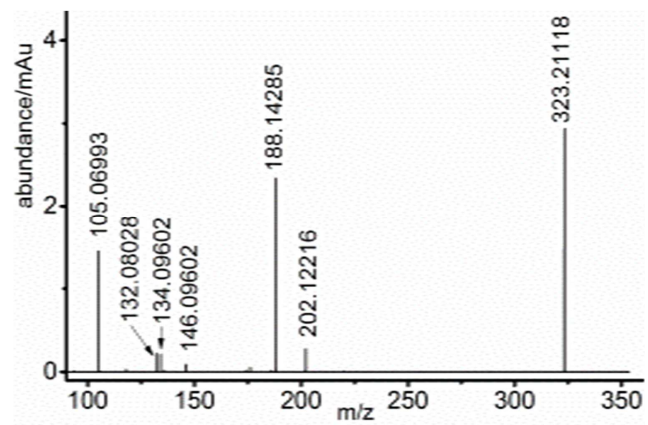

(c)

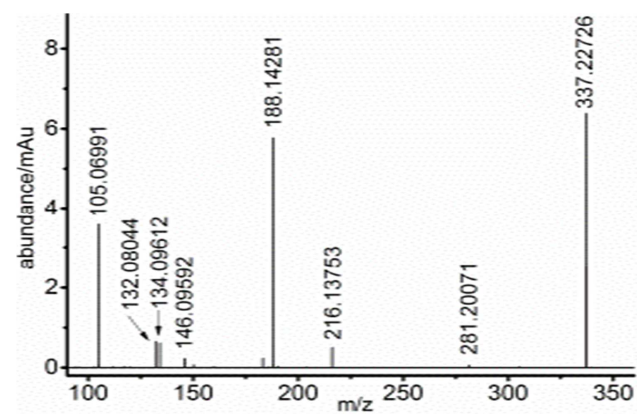

(d)

Figure 3. Secondary ion segments of three target compounds in a positive sample and fentanyl standard (a): 4-ANPP;(b): fentanyl; (c): acetylfentanyl; (d): fentanyl standard.

Table 3. Quasi-molecular ion and secondary ion fragments of three fentanyl substances detected in a positive sample $/ \mathrm{m} / \mathrm{z}$.

\begin{tabular}{|c|c|c|c|c|c|c|c|c|c|c|}
\hline $\begin{array}{l}\text { Peak } \\
\text { No }\end{array}$ & Compound & & {$[\mathbf{M}+\mathbf{H}]$} & $\begin{array}{l}\text { Segment } \\
\text { A }\end{array}$ & $\begin{array}{l}\text { Segment } \\
\text { B }\end{array}$ & $\begin{array}{l}\text { Segment } \\
\text { C }\end{array}$ & $\begin{array}{l}\text { Segment } \\
\text { D }\end{array}$ & $\begin{array}{l}\text { Segment } \\
\text { E }\end{array}$ & $\begin{array}{l}\text { Segment } \\
\text { F }\end{array}$ & $\begin{array}{l}\text { Segment } \\
\text { G }\end{array}$ \\
\hline \multirow{4}{*}{ 1\# } & \multirow{3}{*}{ 4-ANPP } & Theoretical mass & 281.20123 & & 146.09643 & 132.08078 & 105.06988 & 188.14338 & 134.09643 & 160.11208 \\
\hline & & measured mass & 281.20016 & & 146.09605 & 132.08028 & 105.06987 & 188.14275 & 134.09643 & - \\
\hline & & deviation $/ \times 10^{-6}$ & -3.81 & - & -2.60 & -3.79 & -0.10 & -3.35 & 0.00 & - \\
\hline & \multirow{4}{*}{ fentanyl } & Theoretical mass & 337.22744 & 281.20123 & 146.09643 & 132.08078 & 105.06988 & 188.14338 & 134.09643 & 216.13829 \\
\hline \multirow{3}{*}{$2 \#$} & & measured mass & 337.22614 & 281.20007 & 146.09613 & 132.08044 & 105.06991 & 188.14279 & 134.09601 & 216.13757 \\
\hline & & deviation $/ \times 10^{-6}$ & -3.85 & -4.13 & -2.05 & -2.57 & +0.29 & -3.14 & -3.13 & -3.33 \\
\hline & & Theoretical mass & 323.21179 & 281.20123 & 146.09643 & 132.08078 & 105.06988 & 188.14338 & 134.09643 & 202.12264 \\
\hline \multirow{2}{*}{$3 \#$} & \multirow[t]{2}{*}{ acetylfentanyl } & measured mass & 323.21118 & - & 146.09602 & 132.08028 & 105.06993 & 188.14285 & 134.09602 & 202.12216 \\
\hline & & deviation $/ \times 10^{-6}$ & -1.89 & - & -2.81 & -3.79 & +0.48 & -2.82 & -3.06 & -2.38 \\
\hline \multirow[t]{2}{*}{ standard } & fentanyl & measured mass & 337.22726 & 281.20071 & 146.09592 & 132.08044 & 105.06991 & 188.14281 & 134.09612 & 216.13753 \\
\hline & & deviation $/ \times 10^{-6}$ & -0.53 & -1.85 & -3.49 & -2.57 & +0.29 & -3.03 & -2.31 & -3.52 \\
\hline
\end{tabular}

-: segment did not appear

The purity of drugs is of great significance for the sentencing of drug trafficking cases. Therefore, for positive samples, standards are required for purity analysis. Different levels of fentanyl were detected in all 9 samples. Fentanyl standard (purity of 98.5\%) purchased form Alta Scientific Co. Ltd. was analyzed and used to estimate the purity of fentanyl in nine positive samples. The purity of fentanyl was $0.84 \%$ and $1.02 \%$, respectively, for $1 \#$ sample and $2 \#$ sample, in 
which precursor 4-ANPP was the main component. The purity of fentanyl was $85.25 \%, 74.93 \%, 86.42 \%, 79.38 \%, 66.18 \%$, $69.84 \%$ and $59.58 \%$, respectively, for $3 \#$ sample to $9 \#$ sample. Acetylfentanyl and 4-ANPP at trace levels were also detected in these seven positive samples.

\section{Conclusion}

Fentanyl substances are newly regulated psychoactive substances. They are strictly controlled and it is quite difficult to obtain their standards or reference materials. Therefore, it is very difficult to establish an analytical method that can simultaneously detect all 27 controlled fentanyl substances. In this paper, a rapid screening and confirmation method of all 27 controlled fentanyl substances without standards was established based on Orbitrap technology. Rapid screening of the analytes was performed using the accurate mass of the quansi-molecular ion and confirmation was performed utilizing the accurate mass and formula of secondary ion segments. The fentanyl standard was analyzed and the analysis results were compared with those of fentanyl in a positive sample. It was found that the accurate mass deviation of the quasi-molecular ion and the second ion segment between fentanyl standard and fentanyl in a positive sample were all less than 5ppm. Therefore, fentanyl was really detected in a positive sample. The experimental results fully demonstrated the feasibility of the proposed method. The proposed method was simple, rapid, sensitive and accurate, and could be applied to the rapid screening and confirmation of fentanyl-like new psychoactive substances without standard.

\section{References}

[1] Liu Zhimin. Abuse and control of fentanyl and its derivatives: hazards and challenges [J]. Chinese Journal of Drug Dependence, 2017, 26 (4): 274-276.

[2] United Nations Office on Drugs and Crime. What are NPS [EB/OL]. https://www.unodc.org/LSS/Page/NPS, 2016-4-15.

[3] World Health Organization, WHO model list of essential medicines. (2017) [online].

http://www.who.int/medicines/publications/essentialmedicines /en/, 2017-09-22

[4] United States Department of Justice Drug Enforcement Administration. Diversion Control Division National Forensic Laboratory Information System, NFLIS Brief: Fentanyl, 2001-2015, 2017.

[5] I. S. Lurie, A. C. Allen. Reversed-phase high-performance liquid chromatographic separation of fentanyl homologues and analogues [J]. Journal of Chromatography, 1984, 292: 283-294.

[6] R. D. Maier, M. Bogusz. Identification power of a standardized HPLC-DAD system for systematic toxicological analysis [J]. Journal of Analytical Toxicology, 1995, 19 (2): 79-83.

[7] A. A. Almousa, R. Ikeda, M. Wada, et al. HPLC-UV method development for fentanyl determination in rat plasma and its application to elucidate pharmacokinetic behavior after i. p. administration to rats [J]. Journal of Chromato- graphy B: Analytical Technologies in the Biomedical and Life Sciences, 2011, 879 (27): 2941-2944.

[8] T. Naito, Y. Takashina, T. Yagi, et al. Simple and rapid HPLC-UV method using an ultrafine particle octadecylsilane for determination of residual fentanyl in applied durotep MT transdermal matrix patches and its clinical application [J]. Chemical \& Pharmaceutical Bulletin, 2012, 60 (1): 56-61.

[9] Y. Zhang, W. P. Xie. Evaluation of HPLC-DAD selectivity by discrimination power and mean list length for the identification of unknown drugs [J]. Chromatographia, 2014, 77 (23-24): 1613-1622.

[10] H. Ohta, S. Suzuki, K. Ogasawara. Studies on fentanyl and related compounds IV. Chromatographic and spectrometric discrimination of fentanyl and its derivatives [J]. Journal of Analytical Toxicology, 1999, 23: 280-285.

[11] N. F. J. van Nimmen, H. A. F. Veulemans. Validated GC-MS analysis for the determination of residual fentanyl in applied durogesic reservoir durogesic D-trans matrix transdermal fentanyl patches [J]. Journal of Chromatography B: Analytical Technologies in the Biomedical and Life Sciences, 2007, 846 (1-2): 264-272.

[12] P. Kiousi, Y. S. Angelis, E. Lyris, et al. Two-step silylation procedure for the unified analysis of 190 doping control substances in human urine samples by GC-MS [J]. Bioanalysis, 2009, 1 (7): 1209-1224.

[13] B. A. Goldberger, C. W. Chronister, M. L. Merves. Quantitation of fentanyl in blood and urine using gas chromatography-mass spectrometry (GC-MS) [J]. Methods in Molecular Biology, 2010, 603: 245-252.

[14] F. Versace, F. Sporkert, P. Mangin, et al. Rapid sample pre-treatment prior to GC-MS and GC-MS/MS urinary toxicological screening [J]. Talanta, 2012, 101: 299-306.

[15] H. Nair, F. Woo, A. N. Hoofnagle, et al. Clinical validation of a highly sensitive GC-MS platform for routine urine drug screening and real-time reporting of up to 212 drugs [J]. Journal of Toxicology, 2013, 329: 407.

[16] Liu Xiaoyun, Luo Wenguang, Wang Jihua, et al. Study on the qualitative and quantitative analysis method of fentanyl and norfentanyl in human urine by SPE-GC-MS [J]. Chinese Journal of Drug Dependence, 2013, 22 (4): 271-275.

[17] Qian Zhenhua, Li Peng, Zheng Hui, et al. Mass-fragmentation characteristics of fentanyl analogues [J]. Journal of Chinese Mass Spectrometry Society, 2018, 39 (5): 583-592.

[18] M. K. Kioussi, E. M. Lyris, Y. S. Angelis, et al. A generic screening methodology for horse doping control by LC-TOF-MS, GC-HRMS and GC-MS [J]. Journal of Chromatography B: Analytical Technologies in the Biomedical and Life Sciences, 2013, 941: 69-80.

[19] A. H. Wu, R. Gerona, P. Armenian, et al. Role of liquid chromatography-high-resolution mass spectrometry (LC-HR/MS) in clinical toxicology [J]. Clinical Toxicology, 2012, 50: 733-742.

[20] N. N. Stephanson, P. Signell, A. Helander, et al. Use of LC-HRMS in full scan-XIC mode for multi-analyte urine drug testing - a step towards a 'black-box' solution? [J]. Journal of Mass Spectrometry, 2017, 52: 497-506. 
[21] J. L. Manfio, V. J. Santos, V. L. Lanchote, et al Development and validation of an HPLC/MS/MS method for the determination of sufentanil and morphine in human plasma [J]. Journal of AOAC International, 2011, 94 (1): 136-42.

[22] D. M. Bassan, F. Erdmann, R. Kruell. Quantitative determination of 43 common drugs and drugs of abuse in human serum by HPLC-MS/MS [J]. Analytical and Bioanalytical Chemistry, 2011, 400 (1): 43-50.

[23] I. S. Lurie, A. L. Berrier, J. F. Casale, et al. Profiling of illicit fentanyl using UHPLC- MS/MS [J]. Forensic Science International, 2012, 220 (1-3): 191-196.

[24] Y. P. Gaillar, A. C. Cuquel, A. Boucher, et al. A fatality following ingestion of the designer drug meta-chlorophenylpiperazine (mCPP) in an asthmatic-HPLC-MS/MS detection in biofluids and hair [J]. Journal of Forensic Sciences, 2013, 58 (1): 263-269.

[25] D. Remane, D. Montenarh, M. R. Meyer, et al. Application of a UHPLC MS/MS-Based Multianalyte Approach for Screening and Validated Quantification of Drugs in Human Blood Plasma Often Requested in the Context of Brain Death Diagnosis [J]. Therapeutic Drug Monitoring, 2014, 36 (2): 257-260.

[26] S. R. Bista, M. Lobb, A. Haywood, et al. Development, validation and application of an HPLC-MS/MS method for the determination of fentanyl and nor-fentanyl in human plasma and saliva [J]. Journal of Chromatography B: Analytical Technologies in the Biomedical and Life Sciences, 2014, 960: 27-33.

[27] M. E. Blanco, E. Encinas, O. Gonzalez, et al. Quantitative determination of fentanyl in newborn pig plasma and cerebrospinal fluid samples by HPLC-MS/MS [J]. Drug Test Analysis, 2015, 7 (9): 804-811.

[28] X. Wang, S. S. Johansen, M. K. K. Nielsen, et al. Targeted analysis of 116 drugs in hair by UHPLC-MS/MS and its application to forensic cases [J]. Drug Test Analysis, 2017, 9 (8): $1137-1151$

[29] Liu Wei, Xiong Xin, Zhang Xianhua, et al. HPLC-MS/MS method for the determination of sufentanil in human plasma [J] Chinese Journal of Clinical Pharmacology, 2017, 33 (9): 825-827.

[30] K. E. Strayer, H. M. Antonides, M. P. Juhascik, et al. LC-MS/MS-based method for the multiplex detection of 24 fentanyl analogues and metabolites in whole blood at sub ng $\mathrm{mL}^{-1}$ concentrations [J]. ACS Omega, 2018, 3 (1): 514-523.

[31] Kong Lingce, Zou Guomin, Liu Guangqiang, et al.
Surface-enhanced Raman spectroscopy for trace fentanyl detection in water [J]. The Journal of Light Scattering, 2010, 22 (1): 34-38.

[32] F. Inscore, C. Shende, A. Sengupta, et al. Detection of drugs of abuse in saliva by surface-enhanced Raman spectroscopy (SERS) [J]. Appl Spectrosc, 2011, 65 (9): 1004-1008.

[33] P. W. Fedick, B. J. Bills, N. E. Manicke, et al. Forensic sampling and analysis from a single substrate: Surface-enhanced Raman spectroscopy followed by paper spray mass spectrometry [J]. Analytical Chemistry (Washington DC, US), 2017, 89 (20): 10973-10979.

[34] S. Broecker, F. Pragst, A. Bakdash, et al. Combined use of liquid chromatography-hybrid quadrupole time-of-flight mass spectrometry (LC-QTOF-MS) and high performance liquid chromatography with photodiode array detector (HPLC-DAD) in systematic toxicological analysis $[\mathrm{J}]$. Forensic Science International, 2011, 212 (1-3): 215-226.

[35] J. A. Baz-Lomba, M. J. Reid, K. V. Thomas. Target and suspect screening of psychoactive substances in sewage-based samples by UHPLC- QTOF [J]. Analytical Chimica Acta, 2016, 914: 81-90.

[36] J. Aszyk, A. Kot-Wasik. The use of HPLC-Q- TOF-MS for comprehensive screening of drugs and psychoactive substances in hair samples and several "legal highs" products [J]. Monatshefte fur Chemie, 2016, 147 (8): 1407-1414.

[37] D. Chepyala, I. L. Tsai, H. W. Liao, et al. Sensitive screening of abused drugs in dried blood samples using ultra-high-performance liquid chromatography-ion booster-quadrupole time-of- flight mass spectrometry (UHPLC-IB-QTOF-MS) [J]. Journal of Chromatography A, 2017, 1491: 57-66.

[38] Wei Wanli, Dou Li, Zhang Shaoyu, et al. Qualitative and quantitative test of new psychoactive substance methacetin without standard [J]. Journal of People's Public Security University of China (Science and Technology), 2017, (1): 20-26.

[39] C. Noble, D. P. Weihe, J. S. Stybe, et al Application of a screening method for fentanyl and its analogues using UHPLC-QTOF-MS with data-independent acquisition (DIA) in MS (E) mode and retrospective analysis of authentic forensic blood samples [J]. Drug Test Analysis, 2018, 10 (4): 651-662.

[40] E. Jagerdo, J. E. Schaff. Rapid screening for drugs of abuse in biological fluids by ultra high performance liquid chromatography /Orbitrap mass spectrometry [J]. http://dx.doi.org/doi:10.1016/j.jchromb.2016.05.010 